BMJ

Open

Gastroenterology

\title{
Development and validation of a simple and robust model to predict 30-day mortality in patients with Clostridioides difficile-associated enterocolitis
}

\author{
Katrin Claudia Katzer (D) , ${ }^{1}$ Stefan Hagel, ${ }^{2}$ Philipp Alexander Reuken, ${ }^{1}$ \\ Tony Bruns, ${ }^{1,3}$ Andreas Stallmach ${ }^{1}$
}

To cite: Katzer KC, Hagel S, Reuken PA, et al. Development and validation of a simple and robust model to predict 30-day mortality in patients with Clostridioides difficileassociated enterocolitis. BMJ Open Gastro 2020;7:e000468. doi:10.1136/ bmjgast-2020-000468

- Additional material is published online only. To view, please visit the journal online (http://dx.doi.org/10.1136/ bmjgast-2020-000468).

Received 22 June 2020 Revised 2 August 2020 Accepted 13 August 2020
Check for updates

\section{(C) Author(s) (or their} employer(s)) 2020. Re-use permitted under CC BY-NC. No commercial re-use. See rights and permissions. Published by BMJ.

${ }^{1}$ Department of Internal Medicine IV, Jena University Hospital, Jena, Germany ${ }^{2}$ Institute for Infectious Diseases and Infection Control, Jena University Hospital, Jena, Germany

${ }^{3}$ Medical Department III, RWTH Aachen University, Aachen, Nordrhein-Westfalen, Germany

Correspondence to Katrin Claudia Katzer;

Katrin.Katzer@med.uni-jena.de

\section{ABSTRACT}

Objective Clostridioides difficile infection (CDI) is a common healthcare-associated infection and associated with high morbidity and mortality. As current guidelines recommend treatment stratified for disease severity, this study aimed to identify predictors of 30-day mortality in order to develop a robust prediction model.

Design This was a retrospective analysis of 207 inpatients with CDI who were treated at the Jena University Hospital between September 2011 and December 2015. In a training cohort $(n=127)$, predictors of 30-day mortality were identified by receiver operating characteristics analysis and logistic regression. The derived model was validated in an independent cohort of 80 inpatients with CDI.

Results Within 30 days, 35 (28\%) patients in the training cohort died from any cause. C-reactive protein (CRP) of $\geq 121 \mathrm{mg} / \mathrm{L}$ (OR $3.80 ; 95 \% \mathrm{Cl} 1.64$ to $7.80 ; p=0.003$ ) and lower systolic blood pressure of $\leq 104 \mathrm{~mm} \mathrm{Hg}$ (OR 3.73; $95 \% \mathrm{Cl} 1.63$ to 8.53; $\mathrm{p}=0.002$ ) at diagnosis as well as development of renal impairment (serum creatinine $>1.5 \times$ baseline; OR $5.61 ; 95 \% \mathrm{Cl} 1.94$ to 16.26 ; $p=0.035$ ) within the first 6 days were associated with 30-day mortality in univariate analysis. The use of these parameters enabled correct mortality prediction in $73 \%$ of cases on the day of diagnosis and in $76 \%$ at day 6 . In the validation cohort, 30 -day mortality was $18 / 80(23 \%)$. Our model enabled a $73.7 \%$ correct prediction concerning 30-day mortality on day 6 after diagnosis of CDI. Conclusion Hypotension and CRP elevation on the day of diagnosis as well as occurrence of kidney dysfunction during the first 6 days are suitable parameters to predict 30-day mortality in patients with CDI who need to be treated in the hospital.

\section{INTRODUCTION}

Clostridioides difficile is a gram-positive, sporeforming bacterium which is known to cause infectious diarrhoea especially in patients who have recently been treated with antibiotics. ${ }^{1-10}$ Despite the improvement in healthcare facility-associated nosocomial infections, Clostridioides difficile infection (CDI) remains a leading cause of healthcare facility-associated
Summary box

What is already known about this subject?

- Clostridioides difficile infection (CDI) is a common and detrimental healthcare facility-associated infection. Guidelines recommend treatment regimes according to disease severity. Until now, there is no consistent prediction model to identify patients at risk.

What are the new findings?

- Systolic blood pressure and C-reactive protein at diagnosis in addition to the course of serum creatinine allow to identify patients with CDI at risk.

How might it impact on clinical practice in the foreseeable future?

- Our findings allow physicians to identify patients at risk early on during the course of the infection. This allows an adaption of the treatment regime according to the existing guidelines.

infection ${ }^{11} 12$ which results in longer inpatient care $^{13}$ as well as increase in mortality.

CDI varies substantially ranging mild diarrhoea to fulminant disease with high mortality, especially in the elderly or patients with comorbidities. ${ }^{14}$ Over the time, different approaches to stratify disease severity and to identify risk factors for severe disease have been published ${ }^{15-18}$ (online supplementary table 1). However, definitions of severity and prediction models have sometimes been mixed and so far, no model has prevailed in daily practice.

The most commonly used definition of a severe CDI is the one originally published by McDonald et $a l,{ }^{19}$ in which a severe CDI is defined by clinical markers such as necessity to treat the patient in an intensive care unit (ICU) due to CDI or its complications, the need for colectomy due to toxic megacolon or death within 30 days of onset. 
The European Society of Clinical Microbiology and Infectious Diseases (ESCMID) has used this definition as well but modified it by adding prognostic markers including demographic data, blood values and comorbidities. ${ }^{20}$

The guidelines compiled by the Society for Healthcare Epidemiology of America (SHEA) and the Infectious Diseases Society of America differentiate between 'mild or moderate', 'severe' and 'severe and complicated' CDI. They use both laboratory and clinical markers to differentiate between the three. ${ }^{21}$ These guidelines integrate recommendations concerning the different antibiotic regimes based on severity. Those guidelines were renewed in 2017, ${ }^{22}$ with a distinction between first episode, nonsevere; first episode, severe; fulminant disease and recurrent episodes.

The American College of Gastroenterology (ACG) differentiates between 'mild-to moderate', 'severe' and 'severe and complicated' CDI. They too use laboratory and clinical markers and included treatment recommendations according to severity. ${ }^{10}$

In a phase III clinical trial study for fidaxomicin, its safety and efficacy were compared with treatment with vancomycin. Here, a severe case was only defined by unformed stools and blood values. ${ }^{23}$

Lastly, there is a scoring model published by Zar et al which includes age, temperature, blood values, endoscopic evidence of pseudomembranous colitis and necessity to treat the patient in an ICU. ${ }^{24}$

Because there is such a huge variety in the severity of the disease and the current guidelines recommend different antibiotic treatments according to the severity of $\mathrm{CDI},{ }^{1021}$ it is essential to have early prognostic markers to identify patients at risk so the treatment can be adjusted appropriately. Up to now, there is no consistent prediction model for the course of CDI, which makes it difficult for treating physicians to evaluate which treatment regime is suitable for which patient.

As in other infectious diseases, time is crucial for therapeutic success: the sooner a suitable antibiotic treatment regime is initiated, the better the patient's outcome will be. ${ }^{25}$ This was shown for CDI in particular as a therapy according to current guidelines was associated with a decreased risk of mortality. ${ }^{26}$

Therefore, the aim of this retrospective study was to identify and validate prognostic markers for 30-day mortality in two independent cohorts of hospitalised German patients with CDI in order to support severitybased treatment strategies.

\section{METHODS}

\section{Study design}

In order to identify patients with CDI, microbiological data from September 2011 until December 2015 were retrospectively reviewed at the Jena University Hospital. CDI was diagnosed according to ESCMID guidelines. ${ }^{27}$ Day of diagnosis was defined as the day of the stool sample arriving in the lab for testing. All patients with positive results were included if they were treated on an ICU or on a non-intensive internal medicine ward. In patients with recurrent CDI, only the first documented episode of CDI was used for analysis. Patients were allocated to the training (2011-2012) and validation cohort (2013-2015) according to disease onset.

Patients' files, electronic health records, nursing documentation and death certificates were reviewed to identify the following variables: age; gender; living conditions (patient living at home vs patient living in a nursing home vs patient being transferred from another hospital); hospitalisation 3 months prior to diagnosis; surgery 30 days prior to diagnosis; comorbidities (according to the Charlson Comorbidity Index $\left.{ }^{28}\right)$; prior medication; vital parameters (body temperature, heart rate and systolic blood pressure) at diagnosis of CDI; antibiotic therapy including changes in therapy; necessity of treatment on an ICU; need for colectomy due to CDI as well as cause and date of death. In addition, the following laboratory parameters were extracted from our laboratory system: white cell count (WCC), C-reactive protein (CRP), creatinine and albumin. Except for creatinine, which we documented from day of diagnosis daily for the following 7 days, all laboratory markers were only documented on the day of diagnosis, without any scope.

In patients that were discharged before day 30 after CDI diagnosis and who were not treated in our centre again, outcome was assessed by interviewing the general practitioner.

While the definition of a severe case of CDI as published by the SHEA only uses an elevation of serum creatinine $>1.5 \times$ premorbid level ${ }^{21}$ we collected serum creatinine values over the first 7 days after point of diagnosis in order to specify the exact day on which it is possible to identify patients at risk.

Nosocomial infection was defined by criteria used by the Robert Koch Institute (time of diagnosis $>3$ days after admission or inpatient treatment in the last 4 weeks prior to admission) ${ }^{29}$

Additionally, we collected data showing possible indication to an impending systemic inflammatory response syndrome (SIRS). SIRS is defined by two of the following parameters: body temperature $<36^{\circ} \mathrm{C}$ or $>38^{\circ} \mathrm{C}$, heart rate $>90 / \mathrm{min}$, breathing rate $>20 /$ min or $\mathrm{p}_{\mathrm{a}} \mathrm{CO}_{2} \leq 33 \mathrm{~mm}$ $\mathrm{Hg}$, WCC $<4 \times 10^{\wedge} 9 / \mathrm{L}$ or $>12 \times 10^{\wedge} 9 / \mathrm{L}$ or $>10 \%$ immature leucocytes.

Patients consent was waived.

\section{Statistical analysis}

Given the various definitions of CDI severity, we defined 30-day all-cause mortality as the primary end point for the study as the worst possible outcome for any patient. Statistical analyses were performed with SPSS V.22 (IBM).

For comparisons of continuous data, the nonparametric Mann-Whitney $U$ test and for discrete variables the Fisher's exact test was used, respectively. The identification of predictors for 30-day mortality was 
carried out by receiver operating characteristics (ROC) analysis and binary logistic regression. Continuous variables were dichotomised using ROC analysis and Youden index was determined to identify the optimum cut-off. The significance level in two-sided testing was $\mathrm{p}<0.05$. We performed a multivariate logistic regression to develop the prediction model. In backward elimination, a predictor was eliminated if the significance level was $>0.1$. The data are presented as absolute numbers and percentage or as the medians and first and third quartiles.

\section{RESULTS}

\section{Baseline characteristics}

According to the study protocol, 127 patients with CDI were allocated to the training cohort and 100 patients were allocated to the validation cohort. Twenty patients in the validation cohort were lost to follow up and excluded from analysis. Overall, 127 patients from the training cohort and 80 patients from the validation cohort completed follow-up.

In the training cohort, $72(56.7 \%)$ patients were men. Median age of all patients was 74 years (table 1). In the validation cohort, 45 (56.3\%) patients were men; median age was 62 years. One hundred and ten $(86.6 \%)$ and 60 $(75.0 \%)$, respectively, of our cases were nosocomial infections, which is consistent with the previously published articles describing CDI as one of the most common nosocomial infection overall. ${ }^{30}$ Hospitalisation 3 months prior to the diagnosis of CDI was found in $64(50.4 \%)$ patients in our training cohort.

Ninety-four $(74 \%)$ patients of the training cohort were diagnosed while being treated in a normal internal medicine ward, the other 33 (26\%) and patients were being treated in an ICU due to other life-threatening conditions. In the validation cohort, $58(72.5 \%)$ patients were treated in a normal internal medicine ward, 22 (27.5\%) in an ICU.

In our training cohort, the median CRP level on day of diagnosis was $114.3 \mathrm{mg} / \mathrm{L}$ (range $58.3-165.1 \mathrm{mg} / \mathrm{L}$ ), and median WCC was $12.4 \times 10^{\wedge} 9 / \mathrm{L}$ (range $7.6-16.7$ x10^9/L). Median systolic blood pressure was $109 \mathrm{~mm}$ $\mathrm{Hg}$ (range $91-127 \mathrm{~mm} \mathrm{Hg}$ ).

In our validation cohort, the median CRP level on day of diagnosis was $123.9 \mathrm{mg} / \mathrm{L}$ (range $1.9-452.4 \mathrm{mg} / \mathrm{L}$ ). Median systolic blood pressure was $126 \mathrm{~mm} \mathrm{Hg}$ (range 60-166 mm Hg).

On the day of diagnosis, median body temperature was $37.2^{\circ} \mathrm{C}\left(36.0^{\circ} \mathrm{C}-39.8^{\circ} \mathrm{C}\right)$, median heart rate was $84 / \mathrm{min}$ $(78-94 / \mathrm{min})$. We only documented those parameters in our training cohort as did not show any statistical significance with 30-day mortality.

In our training cohort, Charlson Comorbidity Index was $\geq 3$ in $109(85.5 \%)$ patients. Seventy-eight (61.9\%) patients were treated with antibiotics at the time of diagnosis. It was not documented in the reference group since it did not show a statistical significance with 30-day mortality.

\section{Parameters during the course of the infection}

Some authors have previously described an elevation of serum creatinine $>1.5 \times$ baseline to be associated with a severe case of CDI. ${ }^{21}$ In $88(77.2 \%)$ patients of the training cohort, serum creatinine was elevated $>1.5 \times$ baseline level within the first 7 days following the date of diagnosis

In the validation cohort, $67(83.8 \%)$ patients had an elevation of serum creatinine $>1.5 \times$ baseline level within the first 6 days after diagnosis of CDI.

Six $(6.38 \%)$ patients initially treated in a normal internal medicine ward had to be transferred to intermediate or intensive care. One $(0.8 \%)$ patient needed a colectomy due to toxic megacolon as a complication of the CDI. In our training cohort, 35 patients (28\%) died after an average timespan of 10 days (range 1-30) and in our validation cohort, 18 patients $(22.5 \%)$ died after an average timespan of 9 days (range 1-26).

This corresponds with Kaplan-Meier plots which predict a survival of $70.2 \% \pm 4.3 \%$ for the training cohort and $77.8 \% \pm 4.6 \%$ for the validation cohort.

\section{Antibiotic treatment in training cohort}

The most frequently prescribed antibiotic treatment was metronidazole per os, which was prescribed in 72 $(56.7 \%)$ patients (online supplementary table 2 ). Other frequently used antibiotic regimens were vancomycin p.o. (29 patients, $22.8 \%$ ) as well as intravenous metronidazole (12 patients, 9.4\%). A combination of both was given to $7(5.5 \%)$ patients.

Over the course of the infection, in $14(11.0 \%)$ cases, a change of treatment regime was deemed necessary by the treating physicians. This was most common in patients initially treated with metronidazole intravenously $(2 / 11$; $18.2 \%)$. A change was much less frequently necessary in patients treated with metronidazole p.o. $(9 / 71 ; 12.7 \%)$ and vancomycin p.o. (3/28; 10.7\%). Patients initially treated with both vancomycin p.o. and metronidazole intravenously did not need a change of antibiotic treatment in our cohort.

The median time from diagnosis to change in treatment regime was 4 days (range: 1-12 days); the most commonly used antibiotic regime in second line therapy was vancomycin p.o. (6 patients, $42.9 \%$ ).

Empirical antibiotic treatment was associated with 30-day mortality $(\mathrm{p}=0.044)$, therefore underlining the necessity to begin an adequate antibiotic treatment right away. This supports the previously published data and the existing guideline's recommendations.

Our data showed a statistical significance for 30-day mortality concerning the initially chosen therapy $(\mathrm{p}=0.044)$, therefore underlining the necessity to begin an adequate antibiotic treatment right away. This supports the previously published data and the existing guideline's recommendations. In our cohort, there seems to be an advantage for metronidazole concerning survival past 30 days. However, this could be due to sicker 


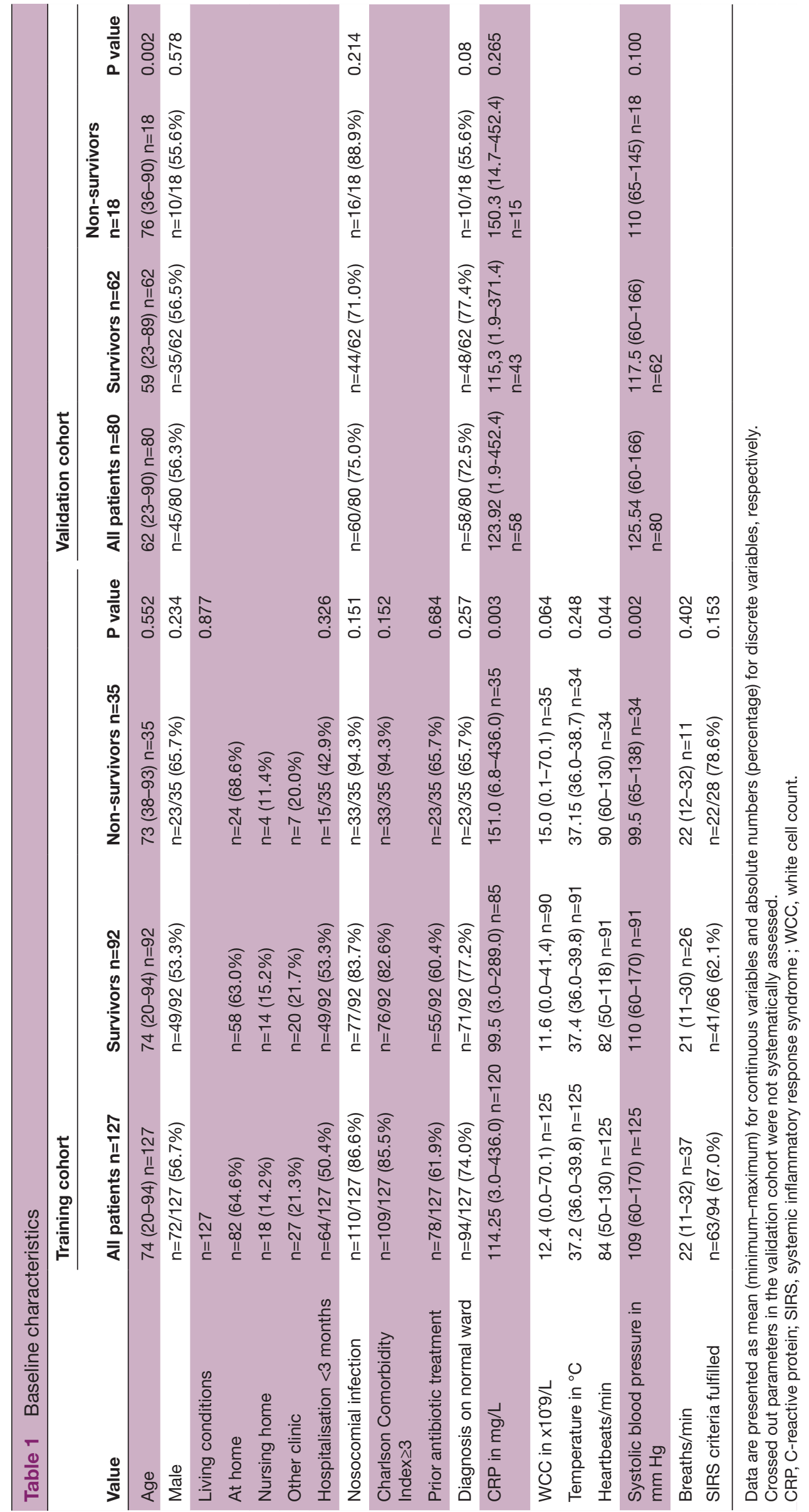


Table 2 Prognostic markers

\begin{tabular}{|c|c|c|c|c|}
\hline \multirow[b]{2}{*}{ Value } & \multicolumn{4}{|l|}{ Training cohort } \\
\hline & All patients $n=127$ & Survivors $\mathrm{n}=92$ & Non-survivors $\mathrm{n}=35$ & $P$ value \\
\hline CRP $\geq 121 \mathrm{mg} / \mathrm{L}$ & $n=55 / 120(45.8 \%)$ & $n=31 / 85(36.5 \%)$ & $n=24 / 35(68.6 \%)$ & 0.002 \\
\hline $\begin{array}{l}\text { Serum creatinine }>1.5 \times \text { baseline within } 6 \\
\text { days after diagnosis }\end{array}$ & $n=18 / 114(15.8 \%)$ & $\mathrm{n}=7 / 82(8.5 \%)$ & $n=11 / 32(34.4 \%)$ & 0.001 \\
\hline
\end{tabular}

Data are presented as absolute numbers (percentage).

CRP, C-reactive protein.

patients receiving vancomycin as first-line therapy as is recommended in the guidelines.

There was no statistical significance concerning 30-day mortality for the change of treatment $(p=1.0)$, the time of change $(\mathrm{p}=0.054)$ or the chosen second-line antibiotic therapy $(\mathrm{p}=0.495)$.

\section{Risk factors}

Parameters which were documented on the day of diagnosis and showed a significant correlation with mortality were elevated CRP (optimal cut-off $\geq 121 \mathrm{mg} / \mathrm{L}$; univariate OR 3.80; 95\% CI 1.64 to $7.80 ; \mathrm{p}=0.003$; sensitivity $=68.6 \%$; specificity $=63.5 \%$; Positive Prospective Value $=43.6 \%$; Negative Prospective Value $=83.1 \%$; Area Under the Curve $=0.675$; table 2 ) as well as low systolic blood pressure (optimal cut-off $\leq 104 \mathrm{~mm} \mathrm{Hg}$; univariate OR 3.73; $95 \%$ CI 1.63 to $8.53 ; \mathrm{p}=0.002$; sensitivity $=64.7 \%$; specificity $=67.0 \%$; Positive Prospective Value $=42.3 \%$; Negative Prospective Value $=83.6 \%$; Area Under the Curve=0.673).

Elevated heart rate at onset also seemed to show a significant correlation with mortality (optimal cut-off $>89$ / min; univariate OR 2.183; $95 \%$ CI 0.979 to 4.866; $\mathrm{p}=0.044$; sensitivity $=58.8 \%$; specificity $=60.4 \%$; Positive Prospective Value $=35.7 \%$; Negative Prospective Value $=79.7 \%$; Area Under the Curve=0.617).

Age, Charlson Comorbidity Index, diagnosis of CDI on ICU, recent surgery and elevated WCC did not show a significant correlation with 30 day mortality.

We performed a multivariate logistic regression involving CRP, heart rate, systolic blood pressure and serum creatinine $>1.5 \times$ baseline within the first 6 days following onset in the training cohort. One hundred and eight patients were enrolled as we had all four variables available. Heart rate on day of diagnosis was excluded due to the significance level (0.463).

Our three remaining risk factors showed an independent association with mortality. A combination of those resulted in a $73.1 \%$ correct prediction of mortality on the day of diagnosis and $75.9 \%$ on day 6 (Area Under the Curve 0.776 ; $95 \%$ CI 0.678 to 0.874 ; sensitivity $=91.2 \%$; specificity $=40.2 \%$; Positive Prospective Value $=66.6 \%$; Negative Prospective Value $=77.4 \%$; Hosmer-Lemeshow test 0.990).

Logistic regression model:
$\mathrm{P}=\frac{\exp (-2.444+0.888 * \mathrm{CRP}+1.348 * \text { systolic blood pressure }+1.797 * \text { creatinine })}{1+\exp (-2.444+0.888 * \mathrm{CRP}+1.348 * \text { systolibloodpressure }+1.797 * \text { creatinine })}$

Enter '0' for CRP/systolic blood pressure/creatinine if the cut-off is not fulfilled, enter ' 1 ' for CRP/systolic blood pressure/creatinine if the cut-off is fulfilled.

High CRP, low systolic blood pressure and increasing creatinine were used as dichotomous variables-either meeting our criteria of CRP $\geq 121 \mathrm{mg} / \mathrm{L}$, systolic blood pressure $\leq 104 \mathrm{~mm} \mathrm{Hg}$ and an elevation of creatinine $>1.5 \times$ baseline (corresponds to ' 1 ' in the model) or not (corresponds to ' 0 ' in the model). ROC analysis exemplify the possibility of differentiating between those patients at risk and those who are not (figure 1A).

In previous publications, immunosuppression, chemotherapy, ongoing antibiotic therapy of underlying disease, elevated serum lactate, treatment with PPI or underlying malignant diseases have been associated with severity of CDI. However, they did not correspond with 30-day mortality in our training cohort (online supplementary table 3).

We validated our derived three-parameter model in an independent cohort of 80 patients. High CRP $\geq 121 \mathrm{mg} / \mathrm{L}$, low systolic blood pressure $\leq 104 \mathrm{~mm} \mathrm{Hg}$ and increased creatinine $>1.5$-fold over baseline resulted in a $73.7 \%$ correct prediction of 30-day mortality at day 6 (Area Under the Curve 0.636; 96\% CI 0.451 to 0.821 ; sensitivity $=46.6 \%$; specificity $=90.2 \%$; Positive Prospective Value $=58.3 \%$; Negative Prospective Value $=82.2 \%$; Hosmer-Lemeshow test 0.927). The performed ROC analysis is shown in (figure 1B).

\section{Comparison with the existing severity definitions}

Among the suggested severity definitions of CDI, only the Zar, Louie and SHEA criteria but not the definitions by ESCMID and ACG were able to discriminate 30-day survivors from non-survivors in the training cohort (table 3 ).

Criteria by Zar et al ( $\mathrm{p}=0.018$; sensitivity $=71.4 \%$; specificity $=52.2 \%$; Positive Prospective Value $=36.2 \%$; Negative Prospective Value $=82.8 \%)$, Louie et al $(\mathrm{p}=0.035$; sensitivity $=48.6 \%$; specificity $=72.2 \%$; Positive Prospective Value $=40.5 \%$; Negative Prospective Value $=78.3 \%$ ) and the SHEA guidelines of $2010(\mathrm{p}=0.001$; sensitivity $=23.5 \%$; specificity $=97.8 \%$; Positive Prospective Value $=80.0 \%$; Negative Prospective Value $=77.0 \%$ ) indicated increased 


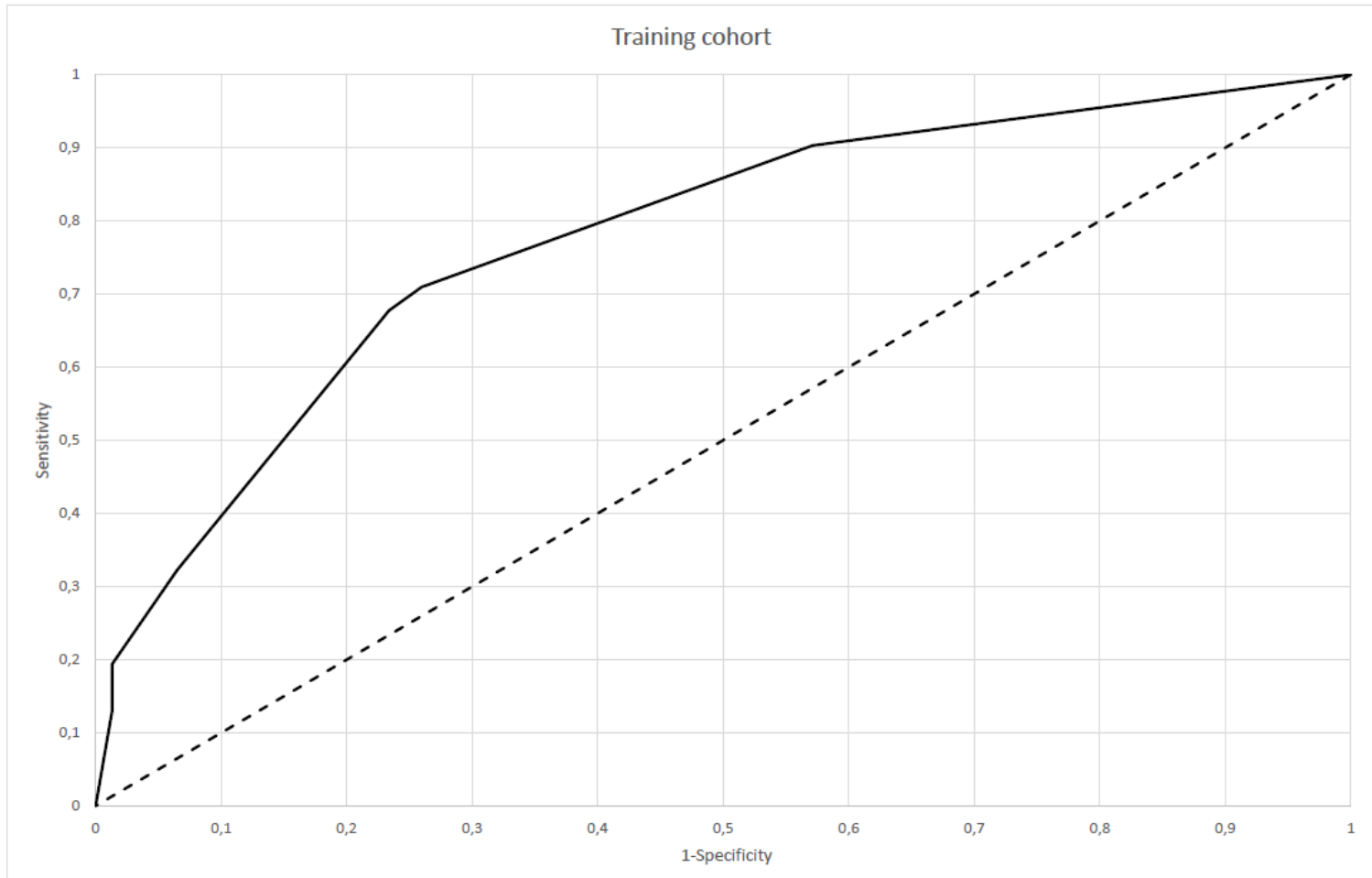

A

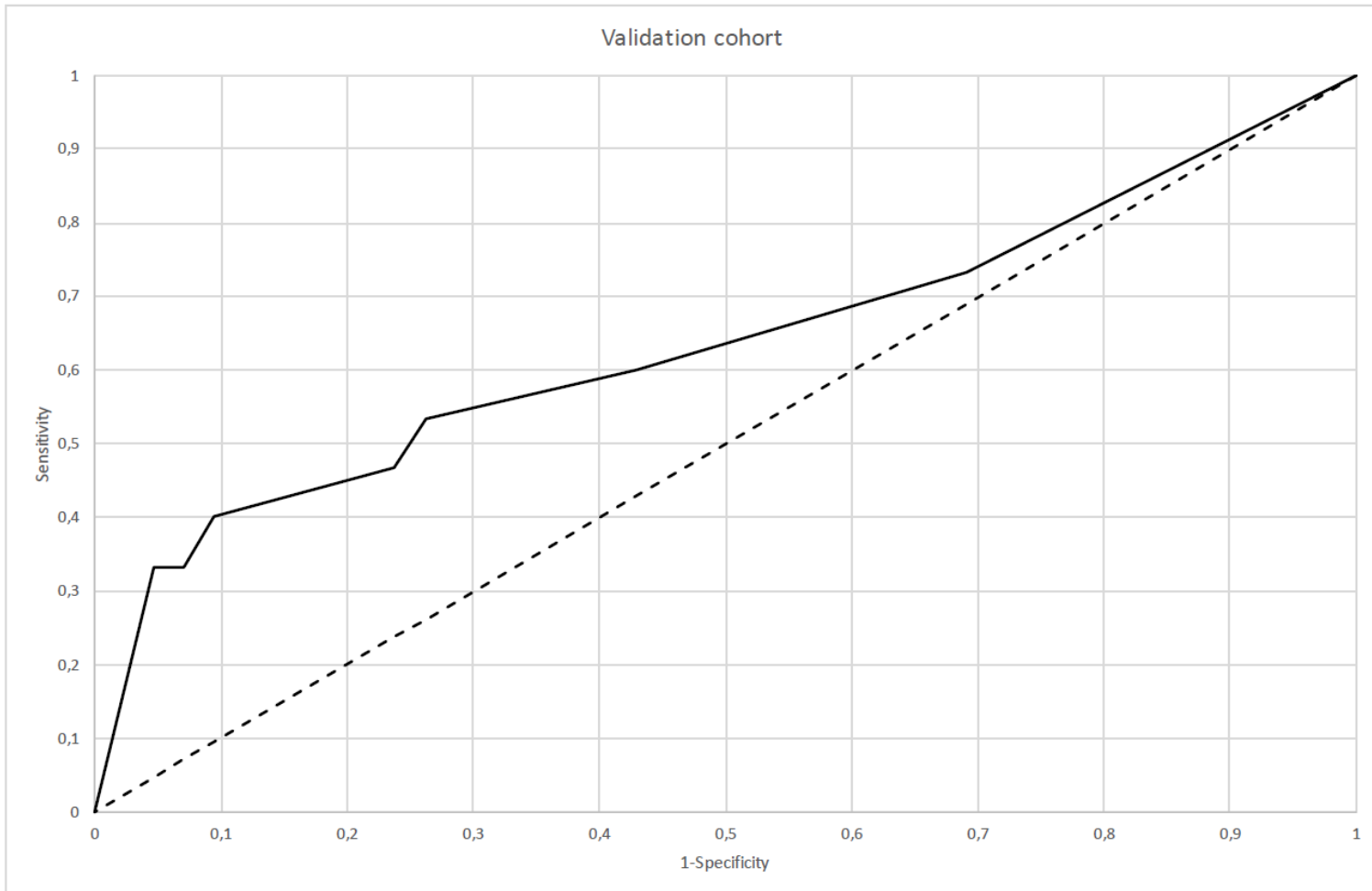

Figure 1 Receiver operating characteristics analysis: (A): in our training cohort and (B) in our validation cohort.

30-day mortality (figure 2). The definitions by ESCMID and ACG did not show any association with 30-day mortality. The patients meeting the criteria of severe disease or fulminant disease according to the SHEA guidelines of 2017 also did not show increased 30-day mortality.

Another established score assessing CDI outcome is the ATLAS score. ${ }^{31}$ Age, treatment with systemic antibiotics 
Table 3 Comparison of the different severity definitions when used on our training cohort

\begin{tabular}{|c|c|c|c|c|}
\hline Value & All patients $n=127$ & Survivors $\mathrm{n}=92$ & Non-survivors $\mathrm{n}=35$ & $P$ value \\
\hline Zar & $n=69 / 127(54.3 \%)$ & $n=44 / 92(47.8 \%)$ & $n=25 / 35(71.4 \%)$ & 0.018 \\
\hline Louie & $n=42 / 125(33.6 \%)$ & $\mathrm{n}=25 / 90(27.8 \%)$ & $\mathrm{n}=17 / 35(48.6 \%)$ & 0.035 \\
\hline ACG severe & $n=23 / 61(37.7 \%)$ & $n=14 / 40(n=35.0 \%)$ & $\mathrm{n}=9 / 21(42.9 \%)$ & 0.587 \\
\hline ACG severe and complicated & $\mathrm{n}=48 / 127(37.8 \%)$ & $n=31 / 92(33.7 \%)$ & $\mathrm{n}=17 / 35(48.6 \%)$ & 0.153 \\
\hline ESCMID & $2(0-4) n=127$ & $2(0-4) n=92$ & $2(0-4) n=35$ & 0.051 \\
\hline SHEA 2010 & $n=10 / 123(8.1 \%)$ & $\mathrm{n}=2 / 89(2.2 \%)$ & $\mathrm{n}=8 / 34(23.5 \%)$ & 0.001 \\
\hline SHEA 2017 & $\mathrm{n}=61 / 83(73.5 \%)$ & $n=38 / 57(66.7 \%)$ & $n=23 / 26(88.5 \%)$ & 0.059 \\
\hline Our model-at least one prediction marker fulfilled & $\mathrm{n}=80 / 116(69.0 \%)$ & $n=49 / 82(59.8 \%)$ & $n=31 / 34(91.2 \%)$ & 0.001 \\
\hline
\end{tabular}

ACG, American College of Gastroenterology; ESCMID, European Society of Clinical Microbiology and Infectious Diseases; SHEA, Society for Healthcare Epidemiology of America.

during CDI, leucocyte count, serum albumin and serum creatinine are part of the score. In our cohort, the patients had an ATLAS score of 5 point in mean (range 2-10) and a higher ATLAS score was not associated with 30-day mortality $(\mathrm{p}=0.290)$.

When using our model in our training cohort, we see a sensitivity of $91.2 \%$ and specificity of $40.2 \%$.

Data are presented as absolute numbers (percentage) of patients who fulfil the criteria of a severe (or severe and complicated) case of CDI. The numbers for the definition by ESCMID data are presented as mean (minimum-maximum).

\section{DISCUSSION}

Considering the increasing incidence, the possible lifethreatening complications and the need for severitystratified treatment, it is crucial to identify patients at risk of mortality. Current guidelines recommend a therapeutic regimen according to severity ${ }^{1021}$ which is associated with better patient outcome. ${ }^{26}{ }^{32}$ However, some criteria for the evaluation of severity, for example, pseudomembrans in endoscopic evaluation or the necessity for ICU therapy, ${ }^{10}{ }^{19}$ are typically not available, respectively, foreseeable at the time of diagnosis. This complicates correct classification and the choice of the adequate antibiotic regimen is difficult in clinical practice.

In this study, we were able to identify CRP levels of $121 \mathrm{mg} / \mathrm{L}$ or higher, systolic blood pressure of $104 \mathrm{~mm}$ $\mathrm{Hg}$ or lower and a more than 1.5-fold increase in creatinine as prognostic markers for 30-day mortality in patients with CDI. The prognostic value could be increased by combining those parameters, showing a correct prediction of $75.9 \%$ of all patient outcomes. Those findings were confirmed in an independent validation cohort.

In the SHEA guidelines, an elevation of serum creatinine above $1.5 \times$ the premorbid level is integrated in the severity classification. In our analysis, we found that the elevation of serum creatinine $>1.5 \times$ at diagnosis of CDI was also associated with an increase in 30-day mortality $(\mathrm{p}<0.0001)$.

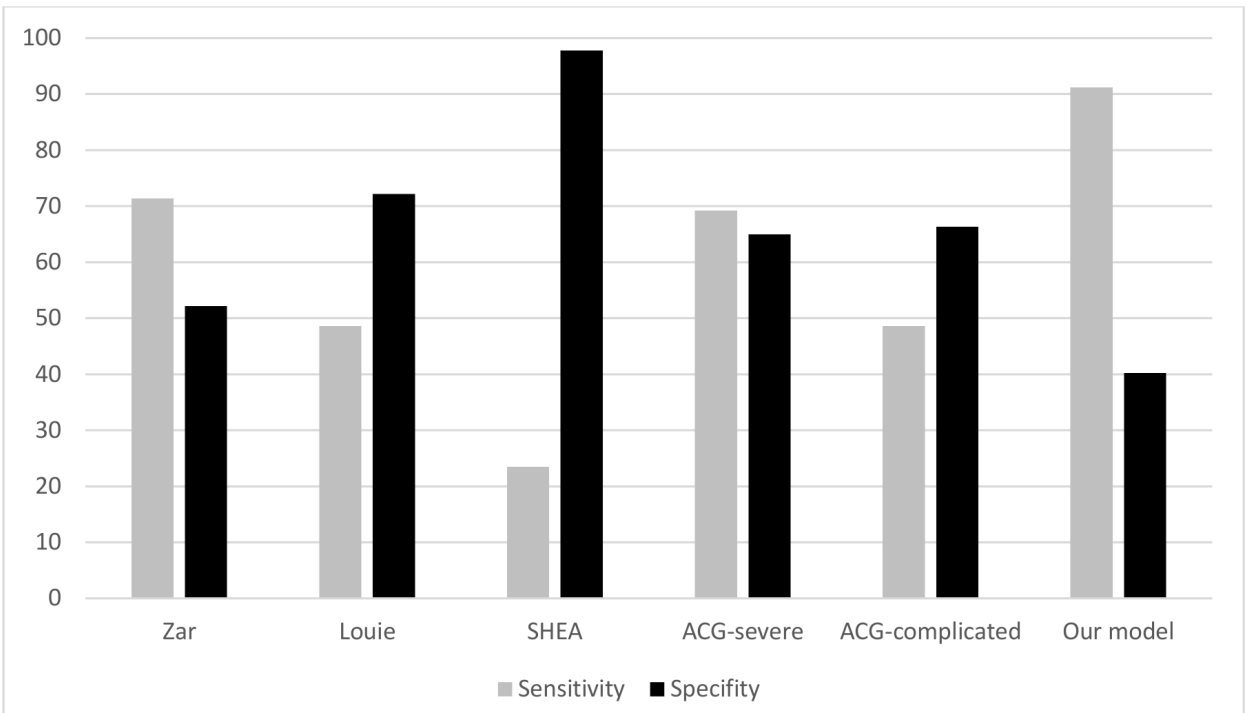

Figure 2 Sensitivity and specificity of the different definitions of severity in our training cohort. ACG, American College of Gastroenterology; SHEA, Society for Healthcare Epidemiology of America. 
All of these parameters, CRP level, systolic blood pressure and baseline serum creatinine, are easy to assess, quickly available and allow a risk-adapted therapy of CDI directly after diagnosis, using CRP level and the systolic blood pressure.

In addition, we were able to show that an elevation of serum creatinine during the first 6 days after diagnosis is a further predictor for a severe course of disease (univariate OR $5.61 ; 95 \%$ CI 1.94 to $16.26 ; \mathrm{p}=0.035$; sensitivity $=34.4 \%$; specificity $=91.5 \%$; Positive Prospective Value $=61.1 \%$; Negative Prospective Value $=78.1 \%$ ). Therefore, monitoring of serum creatinine levels on a regular basis during the first 6 days after diagnosis is also recommended.

In comparison with other existing definitions, predictors and guidelines, our findings allow a risk stratification early on in order to support a physician's decision as to which antibiotic regime is adequate for each individual patient. Furthermore, monitoring of serum creatinine during the first 6 days after day of diagnosis allows ongoing evaluation of the chosen antibiotic regime. Our findings show that the initially chosen antibiotic treatment has an impact on 30-day mortality, underlining the significance of finding the suitable antibiotic treatment for each patient.

As our model presents a significantly higher sensitivity in comparison with the other severity definitions and prediction models, the probability of detecting patient with risk of mortality early on is elevated. On the other hand, our prediction model has a lower specificity than the other severity definitions and prediction models. This will ultimately result in overtherapy for some patients. However, no patient will get harmed by this and patients at risk of mortality will get an adequate treatment early on.

In the before-mentioned severity definitions, there are predictive parameters associated with a severe form of CDI for which we could not find any correlation with 30-day mortality: fever, WCC, low serum albumin, rise in serum lactate, or age did not show any statistical significance in our analysis. We also could not find a difference in 30-day mortality concerning community acquired or nosocomial infection, Charlson Comorbidity Index or inpatient care during the last 30 days prior to admission. ${ }^{5}$

The main limitation is the retrospective nature of the study and the necessity to deal with missing data. Especially, the laboratory parameters were not always documented on the day of diagnosis and are therefore missing. Overall, data were documented better for patients in the ICU as the vital parameters are documented automatically by the monitors and blood withdrawals for the determination of standard values (including serum creatinine) are performed daily. Especially concerning the elevation of serum creatinine, we had to deal with a lot of missing data because the determination of serum creatinine is not regularly done in normal wards on a daily basis. The severity definition by Louie $e t$ alincludes the frequency of unformed stools ${ }^{23}$; this also is often poorly documented and not evaluable in a retrospective analysis. No missing data imputation was performed, and overall, data of 108 patients of the training cohort were used for the multivariable logistic regression model as values of all three predictors were available for them.

Since only data from inpatients were used for this analysis, the model is only validated for hospitalised patients.

Another important factor is that this is a monocentric study. Results could vary in different centres due to local antibiotic stewardship and antibiotic resistance.

Due to the mentioned limitations, our findings need to be evaluated in further prospective multicentric cohorts including both medical and surgical patients as well as ICU patients. Additionally, outpatients should be included in further studies to validate our model for them as well.

Nevertheless, we do think that our results justify using our findings in the daily treatment of patients with CDI. If a patient shows elevated CRP, is hypotensive or develops kidney dysfunction during the course of the infection, we recommend physicians to choose an antibiotic regime according to the current guidelines for patients with severe CDI.

Enter ' 0 ' for CRP/systolic blood pressure/creatinine if the cut-off is not fulfilled, enter ' 1 ' for CRP/systolic blood pressure/creatinine if the cut-off is fulfilled.

Contributors KCK, SH and AS conceived the study. KCK and SH recruited patients and collected the data. KCK and TB performed statistical analysis. KCK and PAR wrote the manuscript. SH, PAR, TB and AS gave intellectual input in data interpretation. All authors read and approved the final version of the manuscript.

Funding The authors have not declared a specific grant for this research from any funding agency in the public, commercial or not-for-profit sectors.

\section{Competing interests None declared.}

Patient consent for publication Not required.

Ethics approval The study protocol was approved by the Internal Review Board (Jena University Hospital Ethics Committee, No. 5428-02/18).

Provenance and peer review Not commissioned; externally peer reviewed.

Data availability statement Data available upon reasonable request from the corresponding author.

Open access This is an open access article distributed in accordance with the Creative Commons Attribution Non Commercial (CC BY-NC 4.0) license, which permits others to distribute, remix, adapt, build upon this work non-commercially, and license their derivative works on different terms, provided the original work is properly cited, appropriate credit is given, any changes made indicated, and the use is non-commercial. See: http://creativecommons.org/licenses/by-nc/4.0/.

\section{ORCID iD}

Katrin Claudia Katzer http://orcid.org/0000-0003-1136-0784

\section{REFERENCES}

1 Bartlett JG, Chang TW, Gurwith M, et al. Antibiotic-Associated pseudomembranous colitis due to toxin-producing clostridia. $N$ Engl J Med 1978;298:531-4.

2 Barbut F, Petit JC. Epidemiology of Clostridium difficile-associated infections. Clin Microbiol Infect 2001;7:405-10.

3 Khanna S, Pardi DS, Aronson SL, et al. The epidemiology of community-acquired Clostridium difficile infection: a populationbased study. Am J Gastroenterol 2012;107:89-95.

4 Hirschhorn LR, Trnka Y, Onderdonk A, et al. Epidemiology of community-acquired Clostridium difficile-associated diarrhea. J infect Dis. 1. Januar 1994;169:127-33. 
5 Clabots CR, Johnson S, Olson MM, et al. Acquisition of Clostridium difficile by hospitalized patients: evidence for colonized new admissions as a source of infection. $J$ Infect Dis 1992;166:561-7.

6 Burdon DW. Clostridium difficile: the epidemiology and prevention of hospital-acquired infection. Infection 1982;10:203-4.

7 Evans CT, Safdar N. Current trends in the epidemiology and outcomes of Clostridium difficile infection. Clin Infect Dis 2015;60 Suppl 2:S66-71.

8 Leffler DA, Lamont JT. Clostridium difficile infection. N Engl J Med 2015;372:1539-48.

9 Vindigni SM, Surawicz CM. C. difficile infection: changing epidemiology and management paradigms. Clin Trans/ Gastroenterol 2015;6:e99.

10 Surawicz CM, Brandt LJ, Binion DG, et al. Guidelines for diagnosis, treatment, and prevention of Clostridium difficile infections. Am J Gastroenterol 2013;108:478-98.

11 McFarland LV. Update on the changing epidemiology of Clostridium difficile-associated disease. Nat Clin Pract Gastroenterol Hepatol 2008:5:40-8.

12 Robert-Koch-Institut. Infektionsepidemiologisches Jahrbuch meldepflichtiger Krankheiten für 2017, 2018. Available: https://www. rki.de/DE/Content/Infekt/Jahrbuch/Jahrbuch_2017.pdf?_blob= publicationFile [Accessed 7 Jan 2020].

13 Dubberke ER, Schaefer E, Reske KA, et al. Attributable inpatient costs of recurrent Clostridium difficile infections. Infect Control Hosp Epidemiol 2014:35:1400-7.

14 Abou Chakra CN, Pepin J, Sirard S, et al. Risk factors for recurrence, complications and mortality in Clostridium difficile infection: a systematic review. PLoS One 2014;9:e98400.

15 Hamo Z, Azrad M, Nitzan O, et al. Role of Single Procalcitonin Test on Admission as a Biomarker for Predicting the Severity of Clostridium difficile Infection. Front Microbiol 2017;8:2532.

16 Brown E, Talbot GH, Axelrod P, et al. Risk factors for Clostridium difficile toxin-associated diarrhea. Infect Control Hosp Epidemiol 1990;11:283-90.

17 Watanakunakorn PW, Watanakunakorn C, Hazy J. Risk factors associated with Clostridium difficile diarrhea in hospitalized adult patients: a case-control study--sucralfate ingestion is not a negative risk factor. Infect Control Hosp Epidemiol 1996;17:232-5.

18 Khanafer N, Barbut F, Eckert C, et al. Factors predictive of severe Clostridium difficile infection depend on the definition used. Anaerobe 2016;37:43-8.

19 McDonald LC, Coignard B, Dubberke E, et al. Recommendations for surveillance of Clostridium difficile-associated disease. Infect Control Hosp Epidemiol 2007;28:140-5.

20 Debast SB, Bauer MP, Kuijper EJ. European Society of clinical microbiology and infectious diseases: update of the treatment guidance document for Clostridium difficile infection. Clinical Microbiology and Infection 2014;20:1-26.

21 Cohen SH, Gerding DN, Johnson S, et al. Clinical practice guidelines for Clostridium difficile infection in adults: 2010 update by the Society for healthcare epidemiology of America (SheA) and the infectious diseases Society of America (IDSA). Infect Control Hosp Epidemiol 2010;31:431-55.

22 McDonald LC, Gerding DN, Johnson S, et al. Clinical practice guidelines for Clostridium difficile infection in adults and children: 2017 update by the infectious diseases Society of America (IDSA) and Society for healthcare epidemiology of America (SheA). Clin Infect Dis 2018;66:e1-48.

23 Louie TJ, Miller MA, Mullane KM, et al. Fidaxomicin versus vancomycin for Clostridium difficile infection. $N$ Engl J Med 2011;364:422-31.

24 Zar FA, Bakkanagari SR, Moorthi KMLST, et al. A comparison of vancomycin and metronidazole for the treatment of Clostridium difficile-associated diarrhea, stratified by disease severity. Clin Infect Dis 2007;45:302-7.

25 Sandiumenge A, Diaz E, Bodí M, et al. Therapy of ventilatorassociated pneumonia. A patient-based approach based on the ten rules of "The Tarragona Strategy". Intensive Care Med 2003;29:876-83.

26 Crowell KT, Julian KG, Katzman M, et al. Compliance with Clostridium difficile treatment guidelines: effect on patient outcomes. Epidemiol Infect 2017;145:2185-92.

27 Bauer MP, Kuijper EJ, van Dissel JT, et al. European Society of clinical microbiology and infectious diseases (ESCMID): treatment guidance document for Clostridium difficile infection (CDI). Clin Microbiol Infect 2009;15:1067-79.

28 Charlson ME, Pompei P, Ales KL, et al. A new method of classifying prognostic comorbidity in longitudinal studies: development and validation. J Chronic Dis 1987;40:373-83.

29 Nationales Referenzzentrum für Surveillance von nosokomialen Infektionen. Definitionen nosokomialer Infektionen für die surveillance Im Krankenhaus-Infektions-Surveillance-System - KISS-Definitionen. Berlin: Robert-Koch-Institut, 2017.

30 Cieślik-Tarkota R, Albertyńska M, Rozwadowska B, et al. Outbreaks of nosocomial infections in Poland in the years 2011-2015. Przegl Epidemiol 2017;71:199-205.

31 Derivation and validation of a simple clinical bedside score (atlas) for Clostridium difficile infection which predicts response to therapy. Available: https://www.ncbi.nlm.nih.gov/pmc/articles/PMC3618004/ [Accessed $20 \mathrm{Jul}$ 2020].

32 Mulherin DW, Hutchison AM, Thomas GJ, et al. Concordance of the SHEA-IDSA severity classification for Clostridium difficile infection and the atlas bedside scoring system in hospitalized adult patients. Infection 2014;42:999-1005. 